\title{
H 2-12, a Misidentified Planetary Nebula in Kepler's SNR
}

\author{
H. Riesgo-Tirado \& J. A. López \\ Instituto de Astronomía UNAM-Ensenada, Apdo. Postal 877, \\ Ensenada, B. C. 22800, México
}

\begin{abstract}
H} \mathrm{2-12}$ (PN 004.5+06.8) is classified as a true or probable planetary nebula (Acker et al. 1992). We obtained narrow-band imagery and long-slit echelle spectra from several positions across $\mathrm{H} 2-12$. It is shown that $\mathrm{H} \mathrm{2-12}$ is associated with one of the brightest condensations located at the periphery of Kepler's SNR. Thus, H 2-12 should be noted as a misclassified PN.
\end{abstract}

\section{H 2-12}

The morphology of $\mathrm{H} \mathrm{2-12}$ is highly peculiar, even for a PN with complex structures. Its main body is formed by a group of distinct knots that follow an elongated and curved morphology. A very extended and remarkably bent filament emerges from the center of the knotty nebulosity, and continues bending towards the north east as if defining a circular structure or cavity which can hardly be identified with a collimated outflow. Neither the line profiles from the knots of $\mathrm{H} \mathrm{2-12}$ show the characteristics usually encountered in $\mathrm{PNe}$, even for those with high-velocity outflows (e.g. López et al. 1997). The kinematics of each knot is characterized by velocity spikes covering a range of $\sim 400$ $\mathrm{km} \mathrm{s}^{-1}$. These peculiar line profiles together with the odd morphology, high $[\mathrm{N} \mathrm{II}] / \mathrm{H} \alpha$ and $[\mathrm{S} \mathrm{II}] / \mathrm{H} \alpha$ line ratios and bright $[\mathrm{O} \mathrm{III}]$ emission are more akin to those expected from a supernova remnant. H 2-12 is not included in the list of misclassified PNe published by Kohoutek $(1994,1997)$ after the previous two IAU symposia on planetary nebulae. We have confirmed that $\mathrm{H} \mathrm{2-12}$ does coincide with the brightest structure on the western side of Kepler's supernova of 1604 and that this nebular, knotty region has indeed been previously associated with this SNR (e.g Bond 1976). Full details of this work will be published elsewhere.

\section{References}

Acker, A. et al. 1992, Stratsbourg-ESO Catalogue of Galactic PNe

Bond, H.E. 1976, PASP, 88, 192

Lopez, J.A., Meaburn, J., Bryce, M. \& Rodriguez, L.F. 1997, ApJ 475, 705

Kohoutek, L. 1994, Astron. Nachr 315, 1, 63

Kohoutek, L. 1997, Astron. Nachr 318, 1, 35 